\title{
OUTER AUTOMORPHISMS OF ORDERED PERMUTATION GROUPS
}

\author{
by W. CHARLES HOLLAND \\ (Received 18th December 1973)
}

\section{Introduction}

A well-known fact is that every automorphism of the symmetric group on a set must be inner (whether the set is finite or infinite) unless the set has exactly six elements $(4, \S 13)$. A long-standing conjecture concerns the analogue of this fact for the group $A(S)$ of all order-preserving permutations of a totally ordered set $S$. The group $A(S)$ is lattice ordered (l-group) by defining, for $f, g \in A(S), f \leqq g$ whenever $x f \leqq x g$ for all $x \in S$. From the standpoint of l-groups, $A(S)$ is of considerable interest because of the analogue of Cayley's theorem proved in (2), namely every $l$-group may be embedded in some $A(S)$. Unlike the non-ordered symmetric groups, which must be highly transitive, $A(S)$ is severely restricted if assumed transitive. Of special interest are those cases when $A(S)$ is doubly transitive (relative to the order), since the building blocks (primitive) of all transitive $A(S)$ are either doubly transitive or uniquely transitive. It is easily seen that each inner automorphism of $A(S)$ must preserve the lattice ordering (that is, must be an l-automorphism). It is tempting to conjecture that every $l$-automorphism of $A(S)$ must be inner. However, an easy counterexample is at hand. If $S$ consists of two copies of the ordered set of integers, one entirely above the other, then $A(S)$ is the direct product of two copies of the $l$-group of integers, which has an outer $l$-automorphism exchanging the two factors. The conjecture referred to above is that if $A(S)$ is transitive on $S$, then every $l$-automorphism of $A(S)$ is inner. The conjecture has been verified for many special classes of groups $A(S)$ satisfying stronger hypotheses than transitivity $((\mathbf{1}),(5),(7),(8))$. In this paper it is shown that the conjecture is false. In fact, there is an example of $A(S)$ which is not only transitive but doubly transitive, and which has an outer $l$-automorphism. Some slightly weaker conjectures are investigated, and it is found that for a transitive $A(S)$, every closed $l$-ideal is $l$-characteristic, and in most, but not all, cases, every $l$-ideal is $l$-characteristic.

\section{A counterexample}

We will construct a totally ordered set $S$ which is doubly homogeneous (for every $x, y, z, w \in S$, if $x<y$ and $z<w$, then there exists $g \in A(S)$ such that $x g=z$ and $y g=w)$ and such that $A(S)$ has an outer $l$-automorphism. I am indebted 
to Andrew Glass for his suggestion to use the ultrafilter in the following construction.

Let $\aleph_{1}$ denote the smallest uncountable ordinal, and for each ordinal $\alpha<\aleph_{1}$, let $Z_{\alpha}=Z$ be the ordered set of integers. Let

$$
R=\prod_{\alpha<N_{1}} Z_{\alpha}=\ldots \times Z_{\alpha} \times \ldots \times Z_{2} \times Z_{1},
$$

which is to be ordered lexicographically from the right. Let $\mathscr{U}$ be an ultrafilter on $\aleph_{1}$ containing all final segments. Let

$$
S=\left\{\left(\ldots, r_{\alpha}, \ldots\right) \in R \mid\left\{\alpha \mid r_{\alpha} \in 2 Z\right\} \in \mathscr{U}\right\} .
$$

Then $S$ is totally ordered as a subset of $R$.

We first show that $A(S)$ is transitive on $S$. Let $\left(\ldots, s_{\alpha}, \ldots\right),\left(\ldots, r_{a}, \ldots\right) \in S$. Then $U=\left\{\alpha \mid r_{\alpha}, s_{\alpha} \in 2 Z\right\} \in \mathscr{U}$. Define $\psi: R \rightarrow R$ by

$$
\left(\ldots, t_{\alpha}, \ldots\right) \psi=\left(\ldots, t_{\alpha}-s_{\alpha}+r_{\alpha}, \ldots\right) \text {. }
$$

Then if $\left(\ldots, t_{\alpha}, \ldots\right) \in S,\left\{\alpha \mid t_{\alpha} \in 2 Z\right\}=V \in \mathscr{U}, \quad V \cap U \in \mathscr{U}$, and for all $\alpha \in V \cap U, t_{\alpha}-s_{\alpha}+r_{\alpha} \in 2 Z$. Hence $\psi: S \rightarrow S$. Similarly $\psi^{-1}: S \rightarrow S$, so that $\psi \in A(S)$. Moreover, $\left(\ldots, s_{\alpha}, \ldots\right) \psi=\left(\ldots, r_{\alpha}, \ldots\right)$.

If $\alpha<\aleph_{1}$, we define an equivalence relation $\equiv_{\alpha}$ on $S$ by

$$
\left(\ldots, r_{\beta}, \ldots\right) \equiv_{\alpha}\left(\ldots, s_{\beta}, \ldots\right)
$$

if $r_{\beta}=s_{\beta}$ for all $\beta \leqq \alpha$. Note that the permutation $\psi$ defined in the previous paragraph respects each of these equivalence relations.

It is easily seen that any one of the equivalence classes $s \equiv_{\alpha}$ is isomorphic to $S$. It follows that any right ray within any equivalence class is isomorphic to any right ray within any other equivalence class (even for different equivalence relations), and the same for left rays.

To show that $A(S)$ is $o$-2-transitive on $S$, it suffices to show that if $x, y, z \in S$ with $x<y<z$, then there exists $g \in A(S)$ such that $x g=x$ and $y g=z$. Let $\alpha$ be the smallest ordinal such that $x \neq_{\alpha} y$. Let $X$ be all lower bounds in $S$ of the $\equiv_{a}$-class containing $y$, and let $Y=S \backslash X$. Then the left ray in the $\equiv_{\alpha}$-class containing $y$ beginning at $y$ is isomorphic to a left ray of $S$. Consequently, $Y$ is isomorphic to $S$. As $A(S)$ is transitive, there exists an order-preserving permutation of $Y$ mapping $y$ to $z$, and we extend this to a permutation of $S$ by mapping $X$ identically onto itself, so the proof is complete.

We note that $S$ is dense in $R$, so we may identify $R$ in an obvious way with a subset of the dedekind completion $\bar{S}$ of $S$. In fact, $R$ is characterised as the points of $\bar{S}$ of lower point character $\aleph_{1}$; that is, for each $r \in R$, the shortest well-ordered subset of $S$ with least upper bound $r$, and not including $r$, has cardinality $\aleph_{1}$. To see that each element of $R$ has this property, suppose that $r=\left(\ldots, r_{\alpha}, \ldots\right) \in R$, and define, for each $\alpha<\aleph_{1}, s_{\alpha}=\left(\ldots, 0, r_{\alpha+1}-1, r_{\alpha}, \ldots, r_{1}\right) \in S$. Then $s_{1}<s_{2}<\ldots<s_{\alpha}<\ldots$, and $r$ is the least upper bound of $\left\{s_{\alpha}\right\}$. No shorter sequence will do, for if $s_{1}^{\prime}<s_{2}^{\prime}<\ldots<r$ is a countable sequence, then letting $d(n)$ be the smallest index at which $s_{n}^{\prime}$ and $r$ differ, $\{d(n) \mid n=1,2, \ldots\}$ is countable 
and so has an upper bound $\beta<\aleph_{1}$. Then the element $s_{\beta}$ defined previously is such that $s_{n}^{\prime}<s_{\beta}<r$, so that $r$ is not the least upper bound of $\left\{s_{n}^{\prime}\right\}$. Conversely, to see that each point of $\bar{S}$ of lower point character $\aleph_{1}$ belongs to $R$, one may consider a bounded increasing sequence of points of $S$,

where

$$
s_{1}<s_{2}<\ldots<s_{\beta}<\ldots, \beta<\aleph_{1} \text {, }
$$

$$
s_{\beta}=\left(\ldots, s_{\beta \alpha}, \ldots\right), \quad \alpha<\aleph_{1} .
$$

An induction argument shows that for each $\alpha$, there exists $\alpha^{\prime}$ such that for all $\beta \geqq \alpha^{\prime}, s_{\beta, \alpha}=s_{a^{\prime}, \alpha^{\prime}}$. The element $r=\left(\ldots, s_{\alpha^{\prime}, \alpha}, \ldots\right)$ of $R$ is then easily seen to be the least upper bound of the sequence $\left\{s_{\beta}\right\}$.

Now we define $\phi: R \rightarrow R$ by $\left(\ldots, x_{\alpha}, \ldots\right) \phi=\left(\ldots, x_{\alpha}+1, \ldots\right)$. Then $\phi$ is an order-preserving permutation of $R$, and $S \phi=R \mid S$ while $S \phi^{2}=S$. It now follows that the mapping $\bar{\phi}=\phi^{-1} g \phi$ is an $l$-automorphism.

The automorphism $\bar{\phi}$ cannot be inner, for if $f \in A(S)$ and $s \in S, s f^{-1} \neq s \phi^{-1}$ since $s f^{-1} \in S$ while $s \phi^{-1} \in R \backslash S$. Suppose, for example, that $s f^{-1}<s \phi^{-1}$. By 0 -2-transitivity, there exists a non-trivial $g \in A(S)$ such that the support of $g$ (supp $g$ ) lies between $s f^{-1}$ and $s \phi^{-1}$. For choose points $x, y \in S$ with $s f^{-1}<x<y<s \phi^{-1}$. Then there exists $1 \leqq g_{1} \in A(S)$ such that $s f^{-1} g_{1}=s f^{-1}$ and $x g_{1}=y$; also there exists $1 \leqq g_{2} \in A(S)$ such that $x g_{2}=y$ and $s \phi^{-1} g_{2}=s \phi^{-1}$; now define $g$ to be the identity off the interval [sf $\left.f^{-1}, s \phi^{-1}\right]$ and $g_{1} \wedge g_{2}$ on this interval. Then $s f^{-1}<\operatorname{supp} g<s \phi^{-1}$. Therefore

$$
\operatorname{supp}\left(\phi^{-1} g \phi\right)<s<\operatorname{supp}\left(f^{-1} g f\right) \text {. }
$$

Hence $g \Phi=\phi^{-1} g \phi \neq f^{-1} g f$, and $\bar{\phi}$ is not inner.

\section{Primitive groups}

Every element of $A(S)$ has a unique extension to an order-preserving permutation of the dedekind completion $\bar{S}$, and we shall make no distinction between the permutation $g \in A(S)$ and its extension.

A fact which is fundamental to all that follows was proved by Lloyd.

Proposition 3.1. (J. T. Lloyd, (5)) Let $A(S)$ be transitive and let every nontrivial interval of $S$ support a non-trivial member of $A(S)$. Then every l-automorphism of $A(S)$ is induced by an inner automorphism of $A(\bar{S})$.

This is precisely the situation of the example in the previous section.

$A(S)$ is said to be an Ohkuma group if it is sharply transitive; that is, for each $x, y \in S$, there exists exactly one $g \in A(S)$ such that $x g=y$. This is clearly the case when $S$ is the set of integers; other less obvious examples are constructed by Ohkuma (9). A congruence is an equivalence relation on $S$, each class of which is convex, and whose classes are permuted by the elements of $A(S) . A(S)$ is o-primitive if the only congruences are the trivial ones. That every Ohkuma group is $o$-primitive follows from (9); also, it is clear that any 
$o$-2-transitive $A(S)$, such as when $S$ is the set of rational numbers or the set of real numbers, is $o$-primitive. For a converse, see Proposition 3.2. An l-ideal of $A(S)$ is a convex normal subgroup sublattice. We note that if $1<g \in A(S)$, the positive $(>1)$ elements of the $l$-ideal of $A(S)$ generated by $g$, are just those positive elements which are bounded above by a product of conjugates of $g$.

We will show that every $l$-ideal of an $o$-primitive $A(S)$ is $l$-characteristic; that is, is mapped onto itself by every $l$-automorphism of $A(S)$.

Proposition 3.2. (See $(\mathbf{1}, \mathbf{5})$.) If $A(S)$ is transitive and o-primitive then either $A(S)$ is an Ohkuma group or $A(S)$ is o-2-transitive.

The following proposition was proved in (2, Theorem 6 ) for the case when $S$ is the chain of real numbers, but the same proof, with minor modifications still works.

Proposition 3.3. Suppose that $A(S)$ is o-2-transitive and that $S$ has a countable coterminal subset (with no upper bound and no lower bound). Then any one of the following four conditions is sufficient to guarantee that there exist conjugates $g^{\prime}$ and $g^{\prime \prime}$ of $g$ such that $f \leqq g^{\prime} \vee g^{\prime \prime}$.

(i) $1<g \in A(S), 1 \leqq f \in A(S)$, and $f$ has bounded support.

(ii) $1<g \in A(S), 1 \leqq f \in A(S)$, the support of $g$ has no upper bound, and the support of $f$ has a lower bound.

(iii) The upper-lower dual of (ii).

(iv) $1<g \in A(S), 1 \leqq f \in A(S)$, and the support of $g$ has neither an upper nor a lower bound.

Corollary 3.4. If $A(S)$ is o-2-transitive and $S$ has a countable coterminal subset, then every l-ideal of $A(S)$ is l-characteristic.

Proof. If $\psi: A(S) \rightarrow A(S)$ is an $l$-automorphism and $1<g \in A(S)$, then Proposition 3.1 implies that the support of $g$ and the support of $g \psi$ have the same " bounded" type. It follows from Proposition 3.3 that

$$
1<g \psi=f \leqq g^{\prime} \vee g^{\prime \prime}
$$

for some conjugates $g^{\prime}$ and $g^{\prime \prime}$ of $g$, and hence that any $l$-ideal containing $g$ must also contain $g \psi$.

It is only a small step now to go to the more general case when $S$ has no countable coterminal subset.

Proposition 3.5. If $A(S)$ is o-2-transitive then every l-ideal of $A(S)$ is $l$ characteristic.

Proof. Let $\psi: A(S) \rightarrow A(S)$ be an $l$-automorphism, induced by the mapping $\bar{\psi}: \bar{S} \rightarrow \bar{S}$ (Proposition 3.1), let $1<g \in A(S)$, and let $T \subseteq \bar{S}$ be the set of common fixed points of $\bar{\psi}$ and $g$. Then $T$ is a closed subset of $\bar{S}$. If $s \in S \mid T$, there exists 
a non-trivial open interval $s^{*}$ of $S$, maximal with respect to the properties $s \in s^{*}$ and $s^{*} \cap T$ is empty. It is obvious that $A\left(s^{*}\right)$ is $o$-2-transitive.

We now show that $s^{*}$ satisfies the hypotheses of Proposition 3.3. Suppose to the contrary, for example, that every countable subset of $s^{*}$ has an upper bound in $s^{*}$. Then since $s \notin T$, either $s g \neq s$ or $s \bar{\psi} \neq s$. The two cases are similar, and we choose the former. Thus $s<s g<s g^{2}<\ldots$ is a countable subset of $s^{*}$ which must have a least upper bound $u_{1} \in \overline{s^{*}}$. It is clear that $u_{1} g=u_{1}$. However, $u_{1} \notin T$, so $u_{1} \Psi \neq u_{1}$. Thus $u_{1}<u_{1}|\bar{\psi}|<u_{1}|\bar{\psi}|^{2}<\ldots$ is a countable subset of $\overline{s^{*}}$ which must have a least upper bound $u_{2} \in \overline{s^{*}}$. Then $u_{2}$ is a fixed point of $\psi$, and hence moved by $g$. Proceeding in the same way, we produce a sequence $u_{1}<u_{2}<\ldots$ of points of $\overline{s^{*}}$ such that each $u_{2 n}$ is a fixed point of $\bar{\psi}$ and each $u_{2 n+1}$ is a fixed point of $g$. This countable sequence must have a least upper bound $u \in \overline{s^{*}}$, and obviously $u$ is fixed both by $g$ and by $\bar{\psi}$. The contradiction establishes that $s^{*}$ does indeed have a countable subset with no upper bound in $s^{*}$. In a dual manner, we see that there is a countable subset with no lower bound in $s^{*}$. Hence $s^{*}$ satisfies the hypotheses of Proposition 3.3.

Because of the definition of $s^{*}, \bar{\psi}$ maps $\bar{s}^{*}$ onto itself, and hence $\bar{\psi}$ induces an $l$-automorphism $\psi^{*}$ of $A\left(s^{*}\right)$. Likewise, if $g^{*}$ is the restriction of $g$ to $s^{*}$, then $g^{*} \in A\left(s^{*}\right)$. Proposition 3.3 now implies that there must exist $h_{1}^{*}, h_{2}^{*} \in A\left(s^{*}\right)$ such that

$$
1<g^{*} \psi^{*} \leqq\left(h_{1}^{*-1} g^{*} h_{1}^{*}\right) \vee\left(h_{2}^{*-1} g^{*} h_{2}^{*}\right)
$$

The construction may be performed on each maximal interval in the complement of $T$ in $\bar{S}$. Finally, we patch together all the various $h_{1}^{*}$ to form $h_{1} \in A(S)$, fixing each point of $T$. Construct $h_{2}$ from the $h_{2}^{*}$ similarly. We then have

$$
1<g \psi \leqq\left(h_{1}^{-1} g h_{1}\right) \vee\left(h_{2}^{-1} g h_{2}\right),
$$

and so every $l$-ideal of $A(S)$ containing $g$ also contains $g \psi$, and the proposition is proved.

It was observed in (1) that an Ohkuma group has no $l$-automorphisms, other than the identity. Hence, the conclusion of Proposition 3.5 is trivially true for Ohkuma groups. Combining this with Proposition 3.2, we have

Theorem 3.6. If $A(S)$ is o-primitive, every l-ideal of $A(S)$ is l-characteristic.

We close this section with the observation that there do exist $o$-primitive examples in which $S$ contains no countable coterminal subset. One such is the "long rational line" which is the lexicographic product $(0,1) \overleftarrow{\times} \aleph_{1}$ where $(0,1)$ is the open rational interval.

\section{Locally Ohkuma groups}

Suppose that $A(S)$ is transitive, yet fails to satisfy the hypothesis of Lloyd's theorem (Proposition 3.1). Then each point $s \in S$ is contained in a minimal interval $O_{s}$ which supports some member of $A(S)$. The sets $O_{s}$ are the classes 
of a congruence on $S$, and $A\left(O_{s}\right)$ is uniquely transitive, hence an Ohkuma group. In this case, we say that $A(S)$ is locally Ohkuma. For example, if $Z$ is the set of integers and $T$ is any homogeneous chain, then $S=Z \overleftarrow{\times} T$ gives rise to a locally Ohkuma $A(S)$ whose congruence classes have the form $Z \times\{t\}, t \in T$. Every locally Ohkuma group arises in this way with $Z$ replaced by some Ohkuma group. Every Ohkuma group is $l$-isomorphic to a subgroup of the real numbers (9).

Let $A(S)$ be locally Ohkuma, and for each $s \in S$ let

$$
L_{s}=\left\{g \in A(S) \mid x g=x, \text { all } x \notin O_{s}\right\} .
$$

Then $L_{s}$ is a convex subgroup of $A(S)$ and is $l$-isomorphic to $A\left(O_{s}\right)$. Hence, like every Ohkuma group, $L_{s}$ is totally ordered. Conversely, let $G \neq\{1\}$ be a totally ordered convex subgroup of $A(S)$. Choose $1<g \in G$ and $s \in S$ such that $s g \neq s$. Then there exists $f \in L_{s}$ such that $s g=s f$. By sharp transitivity, for every $t \in O_{s}, t g=t f$. Hence $1<f \leqq g$, so $f \in G$. Since $L_{s}$ is archimedean, $L_{s} \subseteq G$. Now suppose that $G \neq L_{s}$. Then there exist $1<h \in G, r \notin O_{s}$, such that $r h \neq r$. As before, there exists $1<k \in L_{r} \cap G$. But then $f \wedge k=1$, contradicting the fact that $G$ is totally ordered. Hence $G=L_{s}$. Thus, the groups $L_{s}, s \in S$, are the totally ordered convex subgroups of $A(S)$.

Now let $\bar{\phi}: A(S) \rightarrow A(S)$ be an $l$-automorphism. Choose a reference point $p \in S$. Then $L_{p} \bar{\phi}$ is a totally ordered convex subgroup of $A(S)$, and so $L_{p} \bar{\phi}=L_{q}$ for some $q \in S$. We define a map $\phi: S \rightarrow S$ in the following way. If $s \in S$, there exists $f_{s} \in A(S)$ such that $p f_{s}=s$. Then define $s \phi=q\left(f_{s} \bar{\phi}\right)$. This is independent of the choice of $f_{s}$, for if $p f=p g$, then $f g^{-1}$ fixes each point of $O_{p}$, so that $f g^{-1}$ is disjoint with each element of $L_{p}$. Consequently $\left(f g^{-1}\right) \Phi$ is disjoint with each element of $L_{q}$, so that $f \bar{\phi}$ and $g \bar{\phi}$ agree at each point of $O_{q}$.

To show that $\phi$ is one-to-one and preserves order, let $x, y \in S, x<y$, and choose $f_{x} \in A(S), p f_{x}=x$. Then $p=x f_{x}^{-1}<y f_{x}^{-1}$, and so there exist $1<k \in L_{p}$ and $1 \leqq h \in A(S)$ such that $p k h=y f_{x}^{-1}$. Then

$$
y \phi=q\left(\left(k h f_{x}\right) \bar{\phi}\right)=q(k \bar{\phi})(h \bar{\phi})\left(f_{x} \bar{\phi}\right)>q(h \bar{\phi})\left(f_{x} \bar{\phi}\right) \geqq q\left(f_{x} \bar{\phi}\right)=x \phi,
$$

where the strict inequality occurs because $k \Phi$ belongs to the Ohkuma group $L_{q}$ and so must move every point of $O_{q}$.

It is clear that $\phi$ is onto, and so $\phi \in A(S)$. It is a straight-forward computation to show that for each $g \in A(S), g \Phi=\phi^{-1} g \phi$. This proves

Theorem 4.1. If $A(S)$ is transitive and locally Ohkuma, then every $l$ automorphism of $A(S)$ is inner.

We note that the special case of this theorem when $A(S)$ is locally discrete was proved in (1). Also, we now have a more general form of Lloyd's theorem (Proposition 3.1), without the special " support" hypothesis.

Corollary 4.2. If $A(S)$ is transitive, then every l-automorphism of $A(S)$ is induced by an inner automorphism of $A(\bar{S})$. 


\section{Closed l-ideals}

An $l$-ideal $L$ of a lattice ordered group $G$ is closed if whenever a subset of $L$ has a least upper bound in $G$, that least upper bound belongs to $L$. In this section we will show that every closed $l$-ideal of a transitive $A(S)$ is $l$ characteristic.

The orbits of any $l$-ideal of $A(S)$ are the classes of a congruence. Conversely, if $\mathscr{C}$ is any congruence, $\{g \in A(S) \mid(s g) \mathscr{C} s$, all $s \in S\}$ is a closed $l$-ideal of $A(S)$ (Lloyd (6)), whose orbits are the $\mathscr{C}$-classes. Thus, there is a natural one-to-one correspondence between the closed $l$-ideals of a transitive $A(S)$ and the congruences on $S$. The congruences form a tower under inclusion (1), and hence so do the closed $l$-ideals. It will be convenient to extend each congruence $\mathscr{C}$ on $S$ to a congruence $\overline{\mathscr{C}}$ for the action of $A(S)$ on $\bar{S}$ in the following way. For $x, y \in \bar{S}$ define $x \overline{\mathscr{C}} y$ if $x=y$ or there exist $a, b \in S$ such that $a \mathscr{C} b$ and $x$ and $y$ lie between $a$ and $b$.

Theorem 5.1. If $A(S)$ is transitive, every closed l-ideal of $A(S)$ is $l$ characteristic.

The proof occupies the rest of this section, and proceeds by way of contradiction. Suppose that $A(S)$ is transitive, that $\phi$ is an $l$-automorphism of $A(S)$,

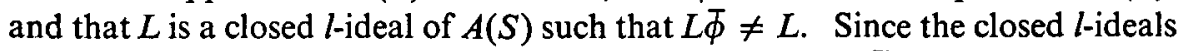
form a tower, we may assume that $L$ is a proper subset of $L \bar{\phi}$. By Corollary 4.2, $\bar{\phi}$ is induced by some $\phi \in A(\bar{S})$. Let $\mathscr{L}^{0}$ be the congruence on $S$ whose classes are the $L$-orbits, and $\overline{\mathscr{L}^{0}}$ its extension to $\bar{S}$ as previously described. Thus, the $\overline{\mathscr{L}^{0}}$-classes are produced by convexifying the $\mathscr{L}^{0}$-orbits in $\bar{S}$. In a similar manner, let $\mathscr{L}^{n}$ and $\overline{\mathscr{L}^{n}}$ denote, respectively, the congruence corresponding to $L \bar{\phi}^{n}$ and its extension.

An easy computation shows

Lemma 5.2. If $\Lambda$ is an $\overline{\mathscr{L}^{n}}$-class, then $\Lambda \phi$ is an $\overline{\mathscr{L}^{n+1}}$-class, and conversely.

Choosing a non-trivial $\overline{\mathscr{L}^{0}}$-class $\Lambda$, by transitivity there exists $g \in A(S)$ such that $\Lambda \bar{\phi} g \supseteq \Lambda$. Replacing $\Phi$ by its product with the inner automorphism induced by $g$, we may assume that $\Lambda$ is a proper subset of $\Lambda \phi$. Then we have, for each integer $n, \Lambda \phi^{n} \subseteq \Lambda^{n+1}$, and each containment is proper.

Let $M$ be the union of all $\Lambda \phi^{n}$. Then $M \phi=M$, so $\phi$ induces an $l$-automorphism of the transitive $A(M)$, under which, the closed $l$-ideal $L^{*}$ corresponding to $L$ is not fixed, and $\cup\left(L^{*} \Phi^{n}\right)=A(M)$. Thus, without loss of generality, we may assume $\cup L \bar{\phi}^{n}=A(S)$.

Let $N=\cap L \bar{\phi}^{n}$. Then $N$ is a closed l-ideal of $A(S)$ and $N \phi=N$. If $\mathcal{N}$ is the corresponding congruence, then $\bar{\phi}$ induces an l-automorphism of $A(S / \mathcal{N})$ under which the closed $l$-ideal $L^{\prime}$ corresponding to $L$ is not fixed, and $\cap\left(L^{\prime} \bar{\phi}^{n}\right)$ is the identity.

Then, without loss of generality, we shall assume for the rest of this proof, E.M.S. $-19 / 4-Z$ 
that $A(S)$ is transitive, $\bar{\phi}$ is an $l$-automorphism of $A(S)$ induced by $\phi \in A(\bar{S})$, $L$ is a closed $l$-ideal of $A(S)$ such that $\cup\left(L \Phi^{n}\right)=A(S)$ and $\cap\left(L \Phi^{n}\right)$ is the identity.

It is easily seen that $\phi$ must permute the orbits of $A(S)$ on $\bar{S}$.

Lemma 5.3. No closed l-ideal of $A(S)$ is fixed by $\bar{\phi}$, except the trivial ones.

Proof. Let $K$ be any non-trivial closed $l$-ideal of $A(S)$. Since the closed l-ideals form a tower and $\cup\left(L \bar{\phi}^{n}\right)=A(S), \cap\left(L \bar{\phi}^{n}\right)=\{1\}$, then for some $n$, $L \phi^{n} \subseteq K \subset L \phi^{n+1}$. It follows that $L \phi^{n+1} \subseteq K \bar{\phi}$, so that $K \neq K \bar{\phi}$.

Lemma 5.4. If for some $x \in \bar{S}, x$ and $x \phi$ lie in the same orbit of $A(S)$ on $\bar{S}$, then that orbit is dense in $\bar{S}$.

Proof. Let $O$ be the orbit containing $x$. Since $\phi$ permutes orbits, and $x \phi \in O, O \phi=O$. Identifying two points of $S$ whenever no point of $O$ lies between them produces a congruence on $S$, whose associated closed $l$-ideal in $A(S)$ is mapped onto itself by $\bar{\phi}$. According to Lemma 5.3, that l-ideal can only be $\{1\}$. It follows that $O$ is dense in $\vec{S}$.

Let

$\Theta=\{x \in \bar{S}|S|$ the orbit of $A(S)$ containing $x$ is dense in $\bar{S}\}$.

Lemma 5.5. $\Theta$ is an orbit of $A(S)$.

Proof. It is obvious that $\Theta A(S)=\Theta$. We now establish that $A(S)$ acts transitively on $\Theta$. Let $x, y \in \Theta$, let $n$ be any integer, and let $\Lambda$ be any $\mathscr{L}^{n}$-class, and let $a, b \in \Lambda, a<b$. Because the orbit of $x$ is dense in $\bar{S}$, there exists $g \in A(S)$ such that $a<x g<b$, so that $x \in \bar{\Lambda} g^{-1}=J_{n}$, a non-trivial $\overline{\mathscr{L}^{n}}$-class. Likewise, $y \in I_{n}$, also a non-trivial $\overline{\mathscr{L}^{n}}$-class. By transitivity again, there exists $g_{n} \in A(S)$ such that $J_{n} g_{n}=I_{n}$.

Let $P_{0}=S \backslash J_{0}$, and for each negative integer $n, P_{n}=J_{n-1} \backslash J_{n}$. Then $\left\{P_{n} \mid n=0,-1,-2, \ldots\right\}$ is a partition of $S$, and we define $f: S \rightarrow S$ by letting $f$ agree with $g_{n}$ on $P_{n}$, for each $n=0,-1,-2, \ldots$ It is easily verified that $f \in A(S)$ and $x f=y$.

Lemma 5.6. For some orbit $O$ of $A(S)$ on $\bar{S}, O \phi=O$.

Proof. We choose $x<y$, points of $S$, such that $x$ and $y$ lie in the same $\mathscr{L}^{n+1}$-class, but not the same $\mathscr{L}^{n}$-class. By Lemma 5.2, $x \phi^{-1}$ and $y \phi^{-1}$ lie in the same $\mathscr{L}^{n}$-class. By transitivity, there exists $g \in A(S)$ such that $x$ and $x \phi^{-1}$ lie in the same $\mathscr{L}^{n}$-class and $x<x \phi^{-1} g$. Then $y \phi^{-1} g$ must also lie in the same $\mathscr{L}^{n}$-class with $x$. Since $y$ lies in a different class,

$$
x<x \phi^{-1} g<y \phi^{-1} g<y \text {. }
$$

Hence the continuous map $\phi^{-1} g: \bar{S} \rightarrow \bar{S}$ must have a fixed point $z \in \bar{S}$, between $x$ and $y$. Thus $z \phi^{-1}=z g^{-1}$; that is, $z$ is mapped by $\phi^{-1}$ into the orbit of $A(S)$ containing $z$. Consequently, that orbit is mapped onto itself by $\phi^{-1}$, and also by $\phi$. 
Lemma 5.7. $\phi \in A(S)$.

Proof. The orbit $O$ of Lemma 5.6 must, according to Lemma 5.4, be dense in $\bar{S}$. It follows from Lemma 5.5 that there are at most two dense orbits, namely $S$ and $\Theta$. If $O=S$, then $S \phi=S$ and we are done. But if $O=\Theta$, then since $S \phi$ must be some dense orbit, and $O \phi=O$, it can only be that $S \phi=S$, and we are done.

The conclusion of Lemma 5.7 is, of course, contradictory, since it implies that $\bar{\phi}$ is inner, while $L \phi \neq L$. Thus we have proved the theorem.

\section{Other $l$-characteristic subgroups}

Proposition 6.1. If $A(S)$ is transitive, if $\Phi$ is an l-automorphism of $A(S)$ induced by the point mapping $\phi \in A(\bar{S})$, and if $\mathscr{C}$ is a non-trivial congruence on $S$, then $\phi$ induces a mapping $\phi^{*}: S / \mathscr{C} \rightarrow S / \mathscr{C}$ so that $\phi$ induces an inner automorphism of the quotient $A(S / \mathscr{C})$.

Proof. Let $L$ be the closed $l$-ideal of $A(S)$ whose orbits are the $\mathscr{C}$-classes, and let $\overline{\mathscr{C}}$ be the natural extension of $\mathscr{C}$ to $\bar{S}$. The non-trivial $\overline{\mathscr{C}}$-classes are precisely those which contain a point of $S$. Moreover, the $\overline{\mathscr{C}}$-classes are the sets obtained by convexifying the orbits of $L$ on $\bar{S}$. By Theorem $5.1, L \bar{\phi}=L$, and so $\phi$ must permute the orbits of $L$ on $\bar{S}$. Hence, $\phi$ permutes the $\mathscr{C}$-classes, and therefore also the non-trivial $\overline{\mathscr{C}}$-classes. The map $\phi^{*}$ is defined, for a $\mathscr{C}$ class $C$, by $C \phi^{*}=D \cap S$ where $D$ is the $\overline{\mathscr{C}}$-class which contains all the points $C \phi$. The permutation $\phi^{*}$ then induces an inner automorphism $\phi^{*}$ of $A(S / \mathscr{C})$. $A(S / \mathscr{C})$ is $l$-isomorphic to $A(S) / L$, and as $L \bar{\phi}=L, \bar{\phi}$ induces an $l$-automorphism of $A(S) / L$, which under the natural identification is the same as $\phi^{*}$.

Lemma 6.2. If $A(S)$ is transitive and not locally Ohkuma, and if $\phi: \bar{S} \rightarrow \bar{S}$ induces an inner automorphism of $A(S)$, then $\phi: S \rightarrow S$.

Proof. As in the paragraph at the end of Section 2, if there exists $s \in S$ such that $s \phi \notin S$, let $f \in A(S)$, so $s \phi \neq s f \in S$. Since $A(S)$ is not locally Ohkuma, every non-trivial interval of $S$ contains the support of a non-trivial member of $A(S)$. Hence there exists $1 \neq g \in A(S)$ whose support lies between $s \phi$ and $s f$. Then clearly $\phi g \phi^{-1} \neq f g f^{-1}$, and hence $\phi$ does not induce an inner automorphism of $A(S)$.

Theorem 6.3. If $A(S)$ is transitive, and for some non-trivial congruence class $C \subseteq S$, every l-automorphism of $A(C)$ is inner, the same is true of $A(S)$.

Proof. In the case that $A(S)$ is locally Ohkuma, the conclusion is true by Theorem 4.1. Suppose $A(S)$ is not locally Ohkuma, and $\phi: \bar{S} \rightarrow \bar{S}$ induces an l-automorphism of $A(S)$. Then if $\vec{C}$ is the dedekind completion of $C$ (the convexification of $C$ in $\bar{S}$ ), $\bar{C} \phi=\bar{D}$, where $D$ is a class of the same congruence, by Proposition 6.1, and so there exists $g \in A(S)$ such that $\phi g: \bar{C} \rightarrow \bar{C}$. Then $\phi g$ induces an $l$-automorphism of $A(C)$, and $A(C)$ cannot be locally Ohkuma 
since $A(S)$ is not. By Lemma 6.2, $\phi g: C \rightarrow C$. Therefore $\phi: C \rightarrow S$. The same is true of every class of the same congruence. Hence $\phi: S \rightarrow S$, so $\phi \in A(S)$.

We now introduce an hypothesis which seems to be slightly stronger than the assumption that every $l$-ideal is $l$-characteristic, although for all we know, it may be equivalent.

If $1<g \in A(S)$ and $\bar{\phi}$ is an l-automorphism of $A(S)$, then $g \bar{\phi} \leqq g^{\prime} g^{\prime \prime}$, where $g^{\prime}$ and $g^{\prime \prime}$ are conjugates of $g$.

The proof of Theorem 3.6 shows that every 0 -primitive $A(S)$ has property $\left({ }^{*}\right)$.

Theorem 6.4. If $A(S)$ is transitive, and for some non-trivial congruence class $C, A(C)$ satisfies $\left({ }^{*}\right)$, then every l-ideal of $A(S)$ is l-characteristic.

Proof. Let $\mathscr{C}$ be the congruence of which $C$ is a class. Let $\bar{\phi}$ be an $l$-automorphism of $A(S)$, and $1<g \in A(S)$. By Proposition 6.1, there exists $f \in A(S)$ such that for each $\mathscr{C}$-class $C, \bar{C} \phi=\bar{C} f$, where $\phi: \bar{S} \rightarrow \bar{S}$ is the mapping which induces $\bar{\phi}$. In particular, $\bar{C}(g \bar{\phi})=\bar{C}(g \bar{f})$. If, for a certain $\bar{C}, \bar{C} f^{-1} g \neq \bar{C} f^{-1}$, then

$$
\bar{C}(g \bar{\phi})=\bar{C} \phi^{-1} g \phi=\bar{C} f^{-1} g f<\bar{C} f^{-1} g^{2} f,
$$

and so certainly for every point $x$ in such a $\bar{C}, x(g \bar{\phi})<x f^{-1} g^{2} f$. In the other case, if $\bar{C} f^{-1} g=\bar{C} f^{-1}$, then if $k$ is the restriction of $f^{-1} g f$ to $C, k \in A(C)$, and $f^{-1} \Phi$ induces an $l$-automorphism of $A(C)$, so by assumption there are two conjugate $k_{C}^{\prime}, k_{C}^{\prime \prime}$ of $k$ such that

$$
g \bar{\phi}=k \bar{f}^{-1} \bar{\phi} \leqq k_{\mathrm{C}}^{\prime} k_{C}^{\prime \prime} \text { on } C .
$$

Of course, $k_{C}^{\prime}$ and $k_{C}^{\prime \prime}$ are also conjugates of $g$. It is easily seen that there exist conjugates $k^{\prime}, k^{\prime \prime}$ of $g$ such that for each $C$ (with $\bar{C} f^{-1} g=\bar{C} f^{-1}$ ), $k^{\prime} \mid C=k_{C}^{\prime}$ and $k^{\prime \prime} \mid C=k_{c}^{\prime \prime}$. Then $1<g \Phi \leqq\left(f^{-1} g f\right) \vee\left(k^{\prime} k^{\prime \prime}\right)$, so $g \Phi$ is in the l-ideal generated by $g$.

Corollary 6.5. If $A(S)$ is transitive and locally o-primitive, every l-ideal of $A(S)$ is l-characteristic.

Let us call $A(S)$ plugged if whenever $\left\{C_{\alpha}\right\}$ is a tower of congruence classes, one from each non-trivial congruence on $S$, then $\cap C_{\alpha}$ is not empty. If $A(S)$ is locally o-primitive and transitive then $A(S)$ is plugged.

Theorem 6.6. If $A(S)$ is transitive, plugged, but not locally o-primitive, then every l-automorphism of $A(S)$ is inner.

Proof. Let $\phi: \bar{S} \rightarrow \bar{S}$ induce an $l$-automorphism of $A(S)$, and let $s \in S$. Then $s=\cap C_{\alpha}$, where the $C_{\alpha}$ are all the non-trivial congruence classes containing $s$. We may also write $s=\cap \overline{C_{\alpha}}$. By Proposition $6.1, \bar{C}_{\alpha} \phi=\bar{D}_{\alpha}$ where $D_{\alpha}$ is a class of the same congruence with $C_{\alpha}$. By assumption, $s \phi=\cap \bar{D}_{\alpha}=\cap D_{\alpha} \in S$. Hence $\phi \in A(S)$. 
If one modifies the example at the end of this section by defining

$$
S=\ldots \times A_{2} \times A_{1},
$$

then $A(S)$ satisfies the hypotheses of Theorem 6.6 .

Finally, we construct an example to show that it is not always the case that for a transitive $A(S)$, every $l$-ideal is $l$-characteristic. By Corollary 6.5 and Theorems 6.3 and 6.6 such an example cannot be plugged and cannot be locally $o$-primitive.

The example will resemble that of Section 2, modified so that the natural equivalence relations become congruences. Let $N$ denote the set of natural numbers. For each $n \in N$, there exists a set $A_{n}$ with the following properties (see (8)). $A_{n}$ is a totally ordered set; $\left|A_{n}\right|=\aleph_{n} ; A_{n}$ has a subset with no upper bound and no lower bound, whose cardinality is $\mathrm{N}_{n-1}$; if $X$ and $Y$ are nonempty subsets of $A_{n}$ such that $|X|,|Y|<\aleph_{n}$ and $X<Y$, then there exists $a \in A_{n}$ with $X<a<Y ; A\left(A_{n}\right)$ is transitive.

Let $\mathbf{Z}$ be the naturally ordered set of integers, and for each $n \in N, T_{n}=\mathbf{Z} \times A_{n}$, ordered lexicographically from the right. Let

$$
E_{n}=\left\{(i, a) \in T_{n} \mid i \text { is even }\right\} .
$$

Let $T=\ldots \times T_{2} \times T_{1}$, ordered lexicographically from the right. Let $\mathscr{U}$ be a non-principal ultrafilter on $N$, and

$$
S=\left\{\left(\ldots, t_{n}, \ldots\right) \in T \mid\left\{n \mid t_{n} \in E_{n}\right\} \in \mathscr{U}\right\} .
$$

We will show that $A(S)$ is transitive and has an $l$-ideal which is not $l$ characteristic.

Suppose that $\left(\ldots, r_{n}, \ldots\right),\left(\ldots, s_{n}, \ldots\right) \in S$. Then since each $A\left(A_{n}\right)$ is transitive, there exists $f_{n} \in A\left(T_{n}\right)$ such that $r_{n} f_{n}=s_{n}$. Moreover, if $r_{n}, s_{n} \in E_{n}$, then $f_{n}$ can be chosen so that $E_{n} f_{n}=E_{n}$. Now define $f: T \rightarrow T$ by $\left(\ldots, t_{n}, \ldots\right) f=\left(\ldots, t_{n} f_{n}, \ldots\right)$. Then $\left(\ldots, r_{n}, \ldots\right) f=\left(\ldots, s_{n}, \ldots\right)$, and if $\left(\ldots, t_{n}, \ldots\right) \in S$, there exists $U \in \mathscr{U}$ such that for all $n \in U, r_{n}, s_{n}, t_{n} \in E_{n}$, which implies $E_{n} f_{n}=E_{n}$, and so $t_{n} f_{n} \in E_{n}$. Hence $\left(\ldots, t_{n}, \ldots\right) f \in S$, so that $f \in A(S)$, showing that $A(S)$ is transitive.

The natural equivalence relations are defined on $S$ by

$$
\left(\ldots, x_{n}, \ldots\right) \equiv_{m}\left(\ldots, y_{n}, \ldots\right)
$$

if $x_{i}=y_{i}$ for all $i \leqq m$. Let $D$ be any $\equiv_{m}$-class. Then $D$ has a certain density property. Namely, let $\left\{a_{\alpha} \mid \alpha<\aleph_{m}\right\}$ be a well-ordered subset of $D$ and $\left\{b_{\alpha} \mid \alpha<\aleph_{m}\right\}$ an inversely well-ordered subset of $D$ with $a_{\alpha}<b_{\beta}$ for all $\alpha, \beta$. Then there exists $n \geqq m$ such that for no $a_{\alpha}, b_{\beta}$ is $a_{\alpha} \equiv_{n+1} b_{\beta}$ (otherwise, the sequences would be countable), but for some $\equiv_{n}$-class $C, C$ contains some $a_{\alpha}$ and some $b_{\beta}$. We now project into $C / \equiv_{n+1}$, which is isomorphic to $T_{n+1}$, producing uncountable sets $\left\{\bar{a}_{\alpha}\right\},\left\{\bar{b}_{\alpha}\right\}$ with $\bar{a}_{\alpha}<\bar{b}_{\beta}$, each $\alpha, \beta$, and $\left|\left\{\bar{a}_{\alpha}\right\}\right|,\left|\left\{\bar{b}_{\alpha}\right\}\right| \leqq \aleph_{m} \leqq \aleph_{n}<\aleph_{n+1}$. It follows that there exists $c \in C$ such that $a_{\alpha}<c<b_{\beta}$, all $\alpha, \beta$.

Now let $f \in A(S)$. If $D f$ were not contained in some one $\equiv_{m}$-class, then $D f$ would contain two points $x, y$ in the same $\equiv_{m-1}$-class $M$ but not in the same 
$\equiv_{m}$-class. Projecting into the quotient $M / \equiv_{m}$, we may assume that $\bar{x}$ and $\bar{y}$ are adjacent points, say $\bar{x}<\bar{y}$ (since $M / \equiv_{m}$ is isomorphic to $T_{m}$ ). $A_{m+1}$, and hence also $T_{m+1}$, has a coterminal subset of cardinality $\aleph_{m}$. If $X$ and $Y$ are, respectively, the $\equiv_{m}$-classes containing $x$ and $y$, the quotient of either by $\equiv_{m+1}$ is isomorphic to $T_{m+1}$, and so there are subsets $\left\{a_{\alpha} \mid \alpha<\aleph_{m}\right\}$ cofinal in $X$ and $\left\{b_{\alpha} \mid \alpha<\aleph_{m}\right\}$ coinitial in $Y$, and since $X$ and $Y$ are adjacent, no element of $D f$ lies between them. This contradicts the fact that $D f$ is isomorphic to $D$. Hence $D f$ is contained in one $\equiv_{m}$-class, and we have proved that each $\equiv_{m}$ is a congruence.

It now follows that $A(S)$ is contained in the wreath product of the groups $A\left(T_{n}\right)$ (3). That is to say, each $g \in A(S)$ is determined by a $N \times S$ matrix $\{g(n, s)\}$ where $g(n, s) \in A\left(T_{n}\right)$ and $\left(\ldots, s_{n}, \ldots\right) g=\left(\ldots, s_{n} g(n, s), \ldots\right)$, and such that if $s \equiv_{n-1} t$, then $g(n, s)=g(n, t)$. The rules of composition, which can easily be deduced are $(f g)(n, s)=f(n, s) g(n, s f)$, and $g^{-1}(n, s)=\left(g\left(n, s g^{-1}\right)\right)^{-1}$.

We will now define a certain element of the wreath product by its matrix components and show it actually belongs to $A(S)$. For each $n \in N$ let $d_{n} \in A\left(T_{n}\right)$ be any non-trivial positive element of bounded support such that $E_{n} d_{n}=E_{n}$. Then define $g(1, s)=1$, and for $n>1$,

$$
g(n, s)=\left\{\begin{array}{l}
1, \text { if } s_{n-1} \in E_{n-1}, \\
d_{n}, \text { otherwise }
\end{array}\right.
$$

The fact that $E_{i} d_{i}=E_{i}$, together with the inversion formula and an induction argument, yield

$$
g^{-1}(n, s)=\left\{\begin{array}{l}
1, \text { if } s_{n-1} \in E_{n-1}, \\
d_{n}^{-1}, \text { otherwise. }
\end{array}\right.
$$

Now let $s \in S$. Then there exists $U \in \mathscr{U}$ such that for all $n \in U, s_{n} \in E_{n}$. Hence for each $n \in U,(s g)_{n}=s_{n} g(n, s)$ which is either $s_{n}$ or $s_{n} d_{n}$, but in either case belongs to $E_{n}$. Therefore $s g \in S$. Similarly, $s g^{-1} \in S$, so $g \in A(S)$.

For each $n \in N$, let $\phi_{n} \in A\left(T_{n}\right)$ be defined by $(i, a) \phi_{n}=(i+1, a)$, and define $\phi \in A(T)$ component-wise by

$$
\phi(n, t)=\phi_{n} \text { for all } t .
$$

It is easily seen that $S \phi=T \backslash S$ and $(T \backslash S) \phi=S$. Noting that $T$ consists of those points of $\bar{S}$ of countable character, and hence $\phi: T \rightarrow T$, it follows that $\phi$ induces an $l$-automorphism $\Phi$ of $A(S)$ by $f \Phi=\phi^{-1} f \phi$. We claim that $g \phi$ does not lie in the $l$-ideal of $A(S)$ generated by $g$, which is, therefore, not $l$-characteristic.

Lemma 6.7. Let $K$ be any finite subset of $A(S)$. Then there exists a point $s \in S$ which is fixed by each $k^{-1} g k, k \in K$, and by $\phi^{-1} g \phi$.

Proof. We define the coordinates of $s$ by induction. Since for $n>1, f(n, s)$ depends not on all coordinates of $s$, but only on $\left(s_{n-1}, \ldots, s_{1}\right)$, we may write $f(n, s)=f\left(n,\left(s_{n-1}, \ldots, s_{1}\right)\right)$. Since $f(1, s)$ is independent of $s$, we may write 
$f(1, s)=f(1,())$. We begin by choosing any $s_{1} \in E_{1}$. Then for every $k \in K \cup\{\phi\}$,

$$
\left(k^{-1} g k\right)(1,())=(k(1,()))^{-1} g(1,()) k(1,(\quad))=1 .
$$

Now suppose we have chosen $s_{1}, \ldots, s_{n}$ such that for each $j \leqq n, s_{j} \in E_{j}$ and for every $k \in K \cup\{\phi\}, s_{j}$ is fixed by $\left(k^{-1} g k\right)\left(j,\left(s_{j-1}, \ldots, s_{1}\right)\right)$. Then

Hence

$$
\left(s_{n}, \ldots, s_{1}\right) k^{-1} g=\left(s_{n}, \ldots, s_{1}\right) k^{-1} \text {. }
$$

$$
\begin{aligned}
& \left(k^{-1} g k\right)\left(n+1,\left(s_{n}, \ldots, s_{1}\right)\right) \\
& \quad=k^{-1}\left(n+1,\left(s_{n}, \ldots, s_{1}\right)\right) g\left(n+1,\left(s_{n}, \ldots, s_{1}\right) k^{-1}\right) k\left(n+1,\left(s_{n}, \ldots, s_{1}\right) k^{-1} g\right) \\
& \quad=\left(k\left(n+1,\left(s_{n}, \ldots, s_{1}\right) k^{-1}\right)\right)^{-1} g\left(n+1,\left(s_{n}, \ldots, s_{1}\right) k^{-1}\right) k\left(n+1,\left(s_{n}, \ldots, s_{1}\right) k^{-1}\right)
\end{aligned}
$$

which has bounded support, since $g\left(n+1,\left(s_{n}, \ldots, s_{1}\right)\right)$ does. Therefore, there exists $s_{n+1} \in E_{n+1}$ which is fixed by each of these. Proceeding by induction, we construct $s=\left(\ldots, s_{2}, s_{1}\right)$ which belongs to $S$ since each $s_{n} \in E_{n}$, and which is fixed by each of the functions $k^{-1} g k, k \in K \cup\{\phi\}$.

Now let $\left\{k_{1}, \ldots, k_{n}\right\} \subseteq A(S), C_{i}=k_{i}^{-1} g k_{i}$, and $p=C_{1} C_{2} \ldots C_{n}$. We will show that $g \bar{\phi} \pm p$. Let $s \in S$ be fixed by each $C_{i}$ and by $\phi^{-1} g \phi$, as guaranteed by the lemma. Then

Also

$$
\begin{aligned}
p(j, s) & =C_{1}(j, s) C_{2}\left(j, s C_{1}\right) \ldots C_{n}\left(j, s C_{1} \ldots C_{n-1}\right) \\
& =C_{1}(j, s) C_{2}(j, s) \ldots C_{n}(j, s) .
\end{aligned}
$$

$$
C_{i}(j, s)=\left(k_{i}\left(j, s k_{i}^{-1}\right)\right)^{-1} g\left(j, s k_{i}^{-1}\right) k_{i}\left(j, s k_{i}^{-1}\right) .
$$

For each $k \in\left\{k_{1}, \ldots, k_{n}\right\}, s k^{-1} \in S$. Therefore, there exists $U \in \mathscr{U}$ such that for all $j \in U$, and for each $k,\left(s k^{-1}\right)_{j} \in E_{j}$, and also $s_{j} \in E_{j}$. Hence, for all $j \in U, p(j+1, s)=1$. On the other hand, for all $j \in U$,

$$
\left(s \phi^{-1}\right)_{j}=s_{j} \phi^{-1}(j, s)=s_{j}\left(\phi\left(j, s \phi^{-1}\right)\right)^{-1} \notin E_{j}, \quad \text { since } s_{j} \in E_{j} .
$$

Hence, $g\left(j+1, s \phi^{-1}\right)=d_{j} \neq 1$, and

$$
(g \phi)(j+1, s)=\left(\phi\left(j+1, s \phi^{-1}\right)\right)^{-1} g\left(j+1, s \phi^{-1}\right) \phi\left(j+1, s \phi^{-1}\right) \neq 1 .
$$

Let $m$ be the smallest integer in $U$. Then since $(g \phi)(m+1, s) \neq 1$, there exists $x \in T_{m+1}$ such that $x(g \bar{\phi})(m+1, s) \neq x$. Let $s^{\prime}$ be any member of $S$ of the form $s^{\prime}=\left(\ldots, x, s_{m}, s_{m-1}, \ldots, s_{1}\right)$. Then

$$
\begin{aligned}
s^{\prime} p & =\left(\ldots, x p(m+1, s), s_{m} p(m, s), \ldots, s_{1} p(1, s)\right) \\
& =\left(\ldots, x, s_{m}, \ldots, s_{1}\right) \\
& <\left(\ldots, x(g \bar{\phi})(m+1, s), s_{m}(g \bar{\phi})(m, s), \ldots, s_{1}(g \bar{\phi})(1, s)\right) \\
& =s^{\prime}(g \bar{\phi}) .
\end{aligned}
$$

Thus, $g \Phi \$ p$. It follows that $g \Phi$ does not belong to the $l$-ideal of $A(S)$ generated by $g$, and so that $l$-ideal is not $l$-characteristic. 


\section{REFERENCES}

(1) W. C. Holland, Transitive lattice ordered permutation groups, Math. $Z$. 87 (1965), 420-433.

(2) W. C. Holland, The lattice ordered group of automorphisms of an ordered set, Michigan Math. J. 10 (1963), 399-408.

(3) W. C. HolLAND and S. H. MCCLEARY, Wreath products of ordered permutation groups, Pacific J. Math. 31 (1969), 703-716.

(4) A. G. Kurosh, The theory of groups (Chelsea, New York, 1960).

(5) J. T. LLoYD, Lattice ordered groups and $o$-permutation groups, $\mathrm{Ph} . \mathrm{D}$. thesis (Tulane University, 1964).

(6) J. T. LLOYD, Complete distributivity in certain infinite permutation groups, Michigan Math. J. 14 (1967), 393-400.

(7) S. H. MCCleary, The closed prime subgroups of certain ordered permutation groups, Pacific J. Math. 31 (1969), 745-753.

(8) S. H. MCCleary, The lattice-ordered group of automorphisms of an $\alpha$-set, Pacific J. Math. 49 (1973), 419-424.

(9) T. OHкuma, Sur quelques ensembles ordonnés linéarment, Fund. Math. 43 (1954), 326:337.

Bowling Green State UnIVERSITy

Bowling Green, OHIO 43403 